\title{
Probabilistic G-contractions
}

\section{Tayyab Kamran ${ }^{1}$, Maria Samreen² and Naseer Shahzad ${ }^{3 *}$}

\section{${ }^{*}$ Correspondence:} nshahzad@kau.edu.sa

${ }^{3}$ Department of Mathematics, King Abdulaziz University, Jeddah, Saudi Arabia

Full list of author information is available at the end of the article

\begin{abstract}
In this paper we introduce the notion of probabilistic G-contraction and establish some fixed point theorems in such settings. Our results generalize/extend some recent results of Jachymski and Sehgal and Bharucha-Reid. Consequently, we obtain fixed point results for $(\epsilon, \delta)$-chainable PM-spaces and for cyclic operators.
\end{abstract}

MSC: $47 \mathrm{H} 10 ; 54 \mathrm{H} 25$

Keywords: fixed point; Menger PM-space; directed graph; Picard operator

\section{Introduction}

In recent years the Banach contraction principle has been widely used to study the existence of solutions for the nonlinear Volterra integral equations, nonlinear integrodifferential equations in Banach spaces and to prove the convergence of algorithms in computational mathematics. It has been extended in many different directions for single- and multi-valued mappings. Recently, Nieto and Rodríguez-López [1], Ran and Reurings [2], Petruşl and Rus [3] established some new results for contractions in partially ordered metric spaces. The following is the main result due to Nieto and Rodríguez-López [1, 4], Ran and Reurings [2].

Theorem 1.1 Let $(S, d)$ be a complete metric space endowed with the partial order ' $\preceq$ '. Assume that the mapping $f: S \rightarrow S$ is nondecreasing (or nonincreasing) with respect to the partial order ' $\leq$ ' on $S$ and there exists a real number $\alpha, 0<\alpha<1$, such that

$$
d(f x, f y) \leq \alpha d(x, y) \text { for all } x, y \in S, x \preceq y .
$$

\section{Also suppose that either}

(i) $f$ is continuous; or

(ii) for every nondecreasing sequence $\left\{x_{n}\right\}$ in $S$ such that $x_{n} \rightarrow x$ in $S$, we have $x_{n} \preceq x$ for all $n \geq 1$.

If there exists $x_{0} \in S$ with $x_{0} \preceq f x_{0}$ (or $x_{0} \succeq f x_{0}$ ), then $f$ has a fixed point. Furthermore, if $(S, \preceq)$ is such that every pair of elements of $S$ has an upper or lower bound, then $f$ is a Picard operator (PO).

Many authors undertook further investigations in this direction to obtain some generalizations and extensions of the above main result (see, e.g., [5-7]). In this context, Jachymski [8] established a generalized and novel version of Theorem 1.1 by utilizing graph theoretic 
approach. From then on, investigations have been carried out to obtain better and generalized versions by weakening contraction condition and analyzing connectivity of a graph (see [9-11]).

Motivated by the work of Jachymski, we can pose a very natural question: Is it possible to establish a probabilistic version of the result of Jachymski [8] (see Corollary 3.12)? In this paper, we give an affirmative answer to this question. Our results are substantial generalizations and improvements of the corresponding results of Jachymski [8] and Sehgal [12] and others (see, e.g., [1, 2, 4]). Subsequently, we apply our main results to the setting of cyclical contractions and to that of $(\epsilon, \delta)$-contractions as well.

\section{Preliminaries}

In 1942 Menger introduced the notion of probabilistic metric space (briefly, PM space), and since then enormous developments in the theory of probabilistic metric space have been made in many directions [13-15]. The fundamental idea of Menger was to replace real numbers with distribution functions as values of a metric.

A mapping $F: \mathbb{R} \rightarrow[0,1]$ is called a distribution function if it is nondecreasing, left continuous and $\inf _{t \in \mathbb{R}} F(t)=0, \sup _{t \in \mathbb{R}} F(t)=1$. In addition, if $F(0)=0$, then $F$ is called a distance distribution function. Let $\mathcal{D}^{+}$denote the set of all distance distribution functions satisfying $\lim _{t \rightarrow \infty} F(t)=1$. The space $\mathcal{D}^{+}$is partially ordered with respect to the usual pointwise ordering of functions, i.e., $F \leq G$ if and only if $F(t) \leq G(t)$ for all $t \in \mathbb{R}$. The element $\epsilon_{0} \in \mathcal{D}^{+}$acts as the maximal element in the space and is defined by

$$
\epsilon_{0}(t)= \begin{cases}0 & \text { if } t \leq 0 \\ 1 & \text { if } t>0\end{cases}
$$

Definition 2.1 A mapping $\Delta:[0,1] \times[0,1] \rightarrow[0,1]$ is called a triangular norm (briefly $t$-norm) if the following conditions hold:

(i) $\Delta$ is associative and commutative,

(ii) $\Delta(a, 1)=a$ for all $a \in[0,1]$,

(iii) $\Delta(a, b) \leq \Delta(c, d)$ for all $a, b, c, d \in[0,1]$ with $a \leq c$ and $b \leq d$.

Typical examples of $t$-norms are $\Delta_{M}(a, b)=\min \{a, b\}$ and $\Delta_{P}(a, b)=a b$.

Definition 2.2 (Hadzić [16], Hadzić and Pap [14]) A $t$-norm $\Delta$ is said to be of $\mathcal{H}$-type if the family of functions $\left\{\Delta^{n}(t)\right\}_{n \in \mathbb{N}}$ is equicontinuous at $t=1$, where $\Delta^{n}:[0,1] \rightarrow[0,1]$ is recursively defined by

$$
\Delta^{1}(t)=\Delta(t, t), \quad \text { and } \quad \Delta^{n}(t)=\Delta\left(\Delta^{n-1}(t), t\right) ; \quad t \in[0,1], \quad n=2,3, \ldots
$$

A trivial example of a $t$-norm of $\mathcal{H}$-type is $\Delta_{M}:=\min$, but there exist $t$-norms of $\mathcal{H}$-type with $\Delta \neq \Delta_{M}$ (see, e.g., [16]).

Definition 2.3 A probabilistic metric space (briefly, PM-space) is an ordered pair $(S, \mathcal{F})$, where $S$ is a nonempty set and $\mathcal{F}: S \times S \rightarrow \mathcal{D}^{+}$if the following conditions are satisfied $\left(\mathcal{F}(p, q)=F_{p, q}, \forall(p, q) \in S \times S\right):$

(PM1) $F_{x, y}(t)=\epsilon_{0}(t) \Longleftrightarrow x=y$ and $x, y \in S$; 
(PM2) $F_{x, y}(t)=F_{y, x}(t)$ for all $x, y \in S$ and $t \in \mathbb{R}$;

(PM3) if $F_{x, y}(t)=1$ and $F_{y, z}(s)=1$, then $F_{x, z}(t+s)=1$ for all $x, y, z \in S$ and for every $t, s \geq 0$.

Definition 2.4 A Menger probabilistic metric space (briefly, Menger PM-space) is a triple $(S, \mathcal{F}, \Delta)$, where $(S, \mathcal{F})$ is a PM-space, $\Delta$ is a $t$-norm and instead of (PM3) in Definition 2.3 it satisfies the following triangle inequality:

$(\mathrm{PM} 3)^{\prime} F_{x, z}(t+s) \geq \Delta\left(F_{x, y}(t), F_{y, z}(s)\right)$ for all $x, y, z \in S$ and $t, s \geq 0$.

Remark 2.5 (Sehgal [12]) Let $(S, d)$ be a metric space. Define $F_{x y}(t)=\epsilon_{0}(t-d(x, y))$ for all $x, y \in S$ and $t>0$. Then the triple $\left(S, \mathcal{F}, \Delta_{M}\right)$ is a Menger PM-space induced by the metric $d$. Furthermore, $\left(S, \mathcal{F}, \Delta_{M}\right)$ is complete iff $d$ is complete.

Schweizer et al. [17] introduced the concept of neighborhood in PM-spaces. For $\varepsilon>0$ and $\delta \in(0,1]$, the $(\varepsilon, \delta)$-neighborhood of $x \in S$ is denoted by $\mathcal{N}_{x}(\varepsilon, \delta)$ and is defined by

$$
\mathcal{N}_{x}(\varepsilon, \delta)=\left\{y \in S: F_{x, y}(\varepsilon)>1-\delta\right\}
$$

Furthermore, if $(S, \mathcal{F}, \Delta)$ is a Menger PM-space with $\sup _{0<a<1} \Delta(a, a)=1$, then the family of neighborhoods $\left\{\mathcal{N}_{x}(\varepsilon, \delta): x \in S, \varepsilon>0, \delta \in(0,1]\right\}$ determines a Hausdorff topology for $S$.

Definition 2.6 Let $(S, \mathcal{F}, \Delta)$ be a Menger PM-space.

(1) A sequence $\left\{x_{n}\right\}$ in $S$ converges to an element $x$ in $S$ (we write $x_{n} \rightarrow x$ or $\left.\lim _{n \rightarrow \infty} x_{n}=x\right)$ if for every $\varepsilon>0$ and $\delta>0$ there exists a natural number $N(\varepsilon, \delta)$ such that $F_{x_{n}, x}(\varepsilon)>1-\delta$, whenever $n \geq N$.

(2) A sequence $\left\{x_{n}\right\}$ in $S$ is a Cauchy sequence if for every $\varepsilon>0$ and $\delta>0$ there exists a natural number $N(\varepsilon, \delta)$ such that $F_{x_{n}, x_{m}}(\varepsilon)>1-\delta$, whenever $n, m \geq N$.

(3) A Menger PM-space is complete if and only if every Cauchy sequence in $S$ converges to a point in $S$.

Now we recall some basic notions from graph theory which we need subsequently. Let $(S, d)$ be a metric space, let $\Omega$ be the diagonal of the Cartesian product $S \times S$, and let $G$ be a directed graph such that the set $V(G)$ of its vertices coincides with $S$ and the set $E(G)$ of its edges contains all loops, i.e., $E(G) \supseteq \Omega$. Assume that $G$ has no parallel edges. Let $G=(V(G), E(G))$ be a directed graph. By letter $\widetilde{G}$ we denote the undirected graph obtained from $G$ by ignoring the direction of edges and by $G^{-1}$ we denote the graph obtained by reversing the direction of edges. Equivalently, the graph $\widetilde{G}$ can be treated as a directed graph having $E(\widetilde{G}):=E(G) \cup E\left(G^{-1}\right)$. If $x$ and $y$ are vertices in a graph $G$, then a path in $G$ from $x$ to $y$ of length $l$ is a sequence $\left(x_{i}\right)_{i=0}^{l}$ of $l+1$ vertices such that $x_{0}=x, x_{l}=y$ and $\left(x_{i-1}, x_{i}\right) \in E(G)$ for $i=1, \ldots, l$. A graph $G$ is called connected if there is a path between any two vertices. $G$ is weakly connected if $\widetilde{G}$ is connected. For a graph $G$ such that $E(G)$ is symmetric and $x$ is a vertex in $G$, the subgraph $G_{x}$ consisting of all edges and vertices which are contained in some path beginning at $x$ is called the component of $G$ containing $x$. In this case $V\left(G_{x}\right)=[x]_{G}$, where $[x]_{G}$ is the equivalence class of a relation $R$ defined on $V(G)$ by the rule: $y R z$ if there is a path in $G$ from $y$ to $z$. Clearly, $G_{x}$ is connected. A mapping $f: S \rightarrow S$ is called a Banach G-contraction [8] if $\forall x, y \in S ;(x, y) \in E(G) \Longrightarrow(f x, f y) \in E(G)$, i.e., $f$ is edge-preserving and $\exists \alpha \in(0,1) \forall x, y \in S((x, y) \in E(G) \Longrightarrow d(f x, f y) \leq \alpha d(x, y))$. 


\section{Main results}

We start with the following definition.

Definition 3.1 A mapping $f: S \rightarrow S$ is said to be a probabilistic G-contraction if $f$ preserves edges and there exists $\alpha \in(0,1)$ such that

$$
\forall x, y \in S, \quad(x, y) \in E(G) \quad \Longrightarrow \quad F_{f x, f y}(\alpha t) \geq F_{x, y}(t)
$$

Example 3.2 Let $(S, d)$ be a metric space endowed with a graph $G$, and let the mapping $f: S \rightarrow S$ be a Banach $G$-contraction. Then the induced Menger PM space $\left(S, \mathcal{F}, \Delta_{M}\right)$ is a probabilistic $G$-contraction.

To see this, let $(x, y) \in E(G)$, then $(f x, f y) \in E(G)$ and there exists $\alpha \in(0,1)$ such that $d(f x, f y) \leq \alpha d(x, y)$. Now, for $t>0$, we have

$$
\begin{aligned}
F_{f x, f y}(\alpha t) & =\epsilon_{0}(\alpha t-d(f x, f y)) \\
& \geq \epsilon_{0}(\alpha t-\alpha d(x, y))=F_{x, y}(t) .
\end{aligned}
$$

Thus $f$ satisfies (3.1).

From Example 3.2 it is inferred that every Banach G-contraction is a probabilistic $G$-contraction with the same contraction constant.

Proposition 3.3 Let $f: S \rightarrow S$ be a probabilistic G-contraction with contraction constant $\alpha \in(0,1)$. Then

(i) $f$ is both a probabilistic $\widetilde{G}$-contraction and a probabilistic $G^{-1}$-contraction with the same contraction constant $\alpha$.

(ii) $\left[x_{0}\right]_{\widetilde{G}}$ is $f$-invariant and $\left.f\right|_{\left[x_{0}\right]_{\widetilde{G}}}$ is a probabilistic $\widetilde{G}_{x_{0}}$-contraction provided that $x_{0} \in S$ is such that $f x_{0} \in\left[x_{0}\right]_{\widetilde{G}}$.

Proof

(i) It follows from the symmetry of $F_{x, y}$.

(ii) Let $x \in\left[x_{0}\right]_{\tilde{G}}$. Then there is a path $x=z_{0}, z_{1}, \ldots, z_{l}=x_{0}$ between $x$ and $x_{0}$. Since $f$ is a probabilistic $G$-contraction, $\left(f z_{i-1}, f z_{i}\right) \in E(G) \forall i=1,2, \ldots, l$. Thus $f x \in\left[f x_{0}\right]_{\tilde{G}}=\left[x_{0}\right]_{\tilde{G}}$. Suppose $(x, y) \in E\left(\tilde{G}_{x_{0}}\right)$. Then $(f x, f y) \in E(G)$ since $f$ is a probabilistic $G$-contraction. But $\left[x_{0}\right]_{\tilde{G}}$ is $f$ invariant, so we conclude that $(f x, f y) \in E\left(\tilde{G}_{x_{0}}\right)$. Condition (3.1) is satisfied automatically, since $\tilde{G}_{x_{0}}$ is a subgraph of $G$.

Lemma 3.4 Let $(S, \mathcal{F}, \Delta)$ be a Menger PM-space under a $t$-norm $\Delta$ satisfying $\sup _{a<1}$ $\Delta(a, a)=1$. Assume that the mapping $f: S \rightarrow S$ is a probabilistic G-contraction. Let $y \in[x]_{\widetilde{G}}$, then $F_{f^{n} f^{\prime} f^{n}}(t) \rightarrow 1$ as $n \rightarrow \infty(t>0)$. Moreover, for $z \in S, f^{n} x \rightarrow z(n \rightarrow \infty)$ if and only iff $f^{n} y \rightarrow z(n \rightarrow \infty)$.

Proof Let $x \in S$ and $y \in[x]_{\widetilde{G}}$, then there exists a path $\left(x_{i}\right), i=0,1,2, \ldots, l$, in $\widetilde{G}$ from $x$ to $y$ with $x_{0}=x, x_{l}=y$ and $\left(x_{i-1}, x_{i}\right) \in E(\widetilde{G})$. From Proposition $3.3, f$ is a probabilistic $\widetilde{G}$-contraction. By induction, for $t>0$, we have $\left(f^{n} x_{i-1}, f^{n} x_{i}\right) \in E(\widetilde{G})$ and $F_{f^{n} x_{i-1}, f^{n} x_{i}}(\alpha t) \geq$ 
$F_{f^{n-1} x_{i-1}, f^{n-1} y_{i}}(t)$ for all $n \in \mathbb{N}$ and $i=1, \ldots, l$. Thus we obtain

$$
\begin{aligned}
F_{f^{n} x_{i-1}, f^{n} x_{i}}(t) & \geq F_{f^{n-1} x_{i-1}, f^{n-1} x_{i}}\left(\frac{t}{\alpha}\right) \\
& \geq F_{f^{n-2} x_{i-1}, f^{n-2} x_{i}}\left(\frac{t}{\alpha^{2}}\right) \\
& \cdots \\
& \geq F_{x_{i-1}, x_{i}}\left(\frac{t}{\alpha^{n}}\right) \rightarrow 1 \quad(\text { as } n \rightarrow \infty) .
\end{aligned}
$$

Let $t>0$ and $\delta>0$ be given. Since $\sup _{a<1} \Delta(a, a)=1$, then there exists $\lambda(\delta) \in(0,1)$ such that $\Delta(1-\lambda, 1-\lambda)>1-\delta$. Choose a natural number $n^{\prime}$ such that for all $n \geq n^{\prime}$ we have $F_{f^{n} x_{0}, f^{n} x_{1}}\left(\frac{t}{2}\right)>1-\lambda$ and $F_{f^{n} x_{1}, f^{n} x_{2}}\left(\frac{t}{2}\right)>1-\lambda$. We get, for all $n \geq n^{\prime}$,

$$
\begin{aligned}
F_{f^{n} x_{0}, f^{n} x_{2}}(t) & \geq \Delta\left(F_{f^{n} x_{0}, f^{n} x_{1}}\left(\frac{t}{2}\right), F_{f^{n} x_{1}, f^{n} x_{2}}\left(\frac{t}{2}\right)\right) \\
& \geq \Delta(1-\lambda, 1-\lambda)>1-\delta,
\end{aligned}
$$

so that $F_{f^{n} x_{0}, f^{n} x_{2}}(t) \rightarrow 1$ as $n \rightarrow \infty(t>0)$. Continuing recursively, one can easily show that

$$
F_{f^{n} x_{0}, f^{n} x_{l}}(t) \rightarrow 1 \quad \text { as } n \rightarrow \infty(t>0) .
$$

Let $f^{n} x \rightarrow z \in S$. Let $t>0$ and $\delta>0$ be given. Since $\sup _{a<1} \Delta(a, a)=1$, then there exists $\lambda(\delta) \in(0,1)$ such that $\Delta(1-\lambda, 1-\lambda)>1-\delta$. Choose a natural number $n_{0}$ such that for all $n \geq n_{0}$ we have $F_{f^{n} x f^{n} y}\left(\frac{t}{2}\right)>1-\lambda$ and $F_{z, f^{n} x}\left(\frac{t}{2}\right)>1-\lambda$. So that for all $n \geq n_{0}$, we have

$$
\begin{aligned}
F_{z, f^{n} y}(t) & \geq \Delta\left(F_{z, f^{n} x}\left(\frac{t}{2}\right), F_{f^{n} x, f^{n_{y}}}\left(\frac{t}{2}\right)\right) \\
& \geq \Delta(1-\lambda, 1-\lambda)>1-\delta .
\end{aligned}
$$

Hence, $f^{n} y \rightarrow z$ as $n \rightarrow \infty$.

Every $t$-norm can be extended in a unique way to an $n$-ary as follows: $\Delta_{i=1}^{0} x_{i}=0, \Delta_{i=1}^{n} x_{i}=$ $\Delta\left(\Delta_{i=1}^{n-1} x_{i}, x_{n}\right)$ for $n=1,2, \ldots$. Let $\left(x_{i}\right)_{i=1}^{l}$ be a path between two vertices $x$ and $y$ in a graph $G$. Let us denote with $L_{x, y}(t)=\Delta_{i=1}^{l} F_{x_{i-1}, x_{i}}(t)$ for all $t$. Clearly the function $L_{x, y}$ is monotone nondecreasing.

Definition 3.5 Let $(S, \mathcal{F}, \Delta)$ be a PM-space and $f: S \rightarrow S$. Suppose that there exists a sequence $\left\{f^{n} x\right\}$ in $S$ such that $f^{n} x \longrightarrow x^{*}$ and $\left(f^{n} x, f^{n+1} x\right) \in E(G)$ for $n \in \mathbb{N}$. We say that:

(i) $G$ is a $\left(C_{f}\right)$-graph in $S$ if there exist a subsequence $\left\{f^{n_{k}} x\right\}$ of $\left\{f^{n} x\right\}$ and a natural number $N$ such that $\left(f^{n_{k}} x, x^{*}\right) \in E(G)$ for $k \geq N$;

(ii) $G$ is an $\left(H_{f}\right)$-graph in $S$ if $f^{n} x \in\left[x^{*}\right]_{\widetilde{G}}$ for $n \geq 1$ and the sequence of functions $\left\{L_{f^{n} x, x^{*}}(t)\right\}$ converges to $\epsilon_{0}(t)$ uniformly as $n \rightarrow \infty(t>0)$.

Example 3.6 Let $(S, \mathcal{F}, \Delta)$ be a Menger PM-space induced by the metric $d(x, y)=|x-y|$ on $S=\left\{\frac{1}{n}: n \in \mathbb{N}\right\} \cup\{0\} \cup \mathbb{N}$, and let $I$ be an identity map on $S$. 
Consider the graph $G_{2}$ consisting of $V\left(G_{2}\right)=S$ and

$$
E\left(G_{2}\right)=\left\{\left(\frac{1}{n}, \frac{1}{n+1}\right),\left(\frac{1}{n+1}, n\right),(n, 0),\left(\frac{1}{5 n}, 0\right) ; n \in \mathbb{N}\right\} .
$$

We note that $x_{n}=\frac{1}{n} \rightarrow 0$ as $n \rightarrow \infty$. Also, it is easy to see that $G_{2}$ is a $\left(C_{I}\right)$-graph. But since $\Delta(a, b)=\min \{a, b\}$, then

$$
\begin{aligned}
L_{x_{n}, 0}(t) & =\Delta\left(\Delta\left(\epsilon_{0}\left(t-\left|\frac{1}{n}-\frac{1}{n+1}\right|\right), \epsilon_{0}\left(t-\left|\frac{1}{n+1}-n\right|\right)\right), \epsilon_{0}(t-n)\right) \\
& =\epsilon_{0}(t-n) \nrightarrow \epsilon_{0}(t) \quad \text { as } n \rightarrow \infty .
\end{aligned}
$$

Thus $G_{2}$ is not an $\left(H_{I}\right)$-graph.

Example 3.7 Let $(S, \mathcal{F}, \Delta)$ be a Menger PM space induced by the metric $d(x, y)=|x-y|$ on $S=\left\{\frac{1}{n}: n \in \mathbb{N}\right\} \cup\left\{\frac{\sqrt{5}}{n+1}: n \in \mathbb{N}\right\} \cup\{0\}$, and let $I$ be an identity map on $S$. Consider the graph $G_{3}$ consisting of $V\left(G_{3}\right)=S$ and

$$
E\left(G_{3}\right)=\left\{\left(\frac{1}{n}, \frac{1}{n+1}\right),\left(\frac{1}{n}, \frac{\sqrt{5}}{n+1}\right),\left(\frac{\sqrt{5}}{n+1}, 0\right) ; n \in \mathbb{N}\right\} .
$$

Since $x_{n}=\frac{1}{n} \rightarrow 0$ as $n \rightarrow \infty$. Clearly, $G_{4}$ is not a $\left(C_{I}\right)$-graph. But $L_{x_{n}, 0}(t)=\epsilon_{0}\left(t-\frac{\sqrt{5}}{n+1}\right) \rightarrow$ $\epsilon_{0}(t)$ as $n \rightarrow \infty(t>0)$. Thus $G_{3}$ is an $\left(H_{I}\right)$-graph.

From the above examples, we note that the notions of $\left(C_{f}\right)$-graph and $\left(H_{f}\right)$-graph are independent even if $f$ is an identity map.

The following lemma is essential to prove our fixed point results.

Lemma 3.8 (Miheț [18]) Let $(S, \mathcal{F}, \Delta)$ be a Menger PM-space under a t-norm $\Delta$ of H-type. Let $\left\{x_{n}\right\}$ be a sequence in $S$, and let there exist $\alpha \in(0,1)$ such that

$$
F_{x_{n}, x_{n+1}}(\alpha t) \geq F_{x_{n-1}, x_{n}}(t) \quad \text { for all } n \in \mathbb{N}, t>0 \text {. }
$$

Then $\left\{x_{n}\right\}$ is a Cauchy sequence.

Theorem 3.9 Let $(S, \mathcal{F}, \Delta)$ be a complete Menger PM-space under a t-norm $\Delta$ of $\mathcal{H}$-type. Assume that the mapping $f: S \rightarrow S$ is a probabilistic G-contraction and there exists $x_{0} \in S$ such that $\left(x_{0}, f x_{0}\right) \in E(G)$, then the following assertions hold.

(i) If $G$ is a $\left(C_{f}\right)$-graph, then $f$ has a unique fixed point $\varrho \in\left[x_{0}\right]_{\tilde{G}}$ and for any $y \in\left[x_{0}\right]_{\widetilde{G}}$, $f^{n} y \rightarrow \varrho$. Moreover, if $G$ is weakly connected, then $f$ is a Picard operator.

(ii) If $G$ is a weakly connected $\left(H_{f}\right)$-graph, then $f$ is a Picard operator.

Proof Since $f$ is a probabilistic G-contraction and there exists $x_{0} \in S$ such that $\left(x_{0}, f x_{0}\right) \in$ $E(G)$. By induction $\left(f^{n} x_{0}, f^{n+1} x_{0}\right) \in E(G)$ for all $n \geq 1$ and

$$
F_{f^{n} x_{0}, f^{n+1} x_{0}}(\alpha t) \geq F_{f^{n-1} x_{0}, f^{n} x_{0}}(t) \quad \text { for all } n \geq 1 .
$$


(i) Since the $t$-norm $\Delta$ is of $\mathcal{H}$-type, then from Lemma 3.8 it can be inferred that $\left\{f^{n} x_{0}\right\}$ is a Cauchy sequence in $S$. From completeness of the Menger PM-space $S$, there exists $\varrho \in S$ such that

$$
\lim _{n \rightarrow \infty} f^{n} x_{0}=\varrho
$$

Now we prove that $\varrho$ is a fixed point of $f$. Let $G$ be a $\left(C_{f}\right)$-graph. Then there exists a subsequence $\left\{f^{n_{k}} x_{0}\right\}$ of $\left\{f^{n} x_{0}\right\}$ and $N \in \mathbb{N}$ such that $\left(f^{n_{k}} x_{0}, \varrho\right) \in E(G)$ for all $k \geq N$. Note that $\left(x_{0}, f x_{0}, f^{2} x_{0}, \ldots, f^{n_{1}} x_{0}, \ldots, f^{n_{N}} x_{0}, \varrho\right)$ is a path in $G$ and so in $\widetilde{G}$ from $x_{0}$ to $\varrho$, thus $\varrho \in\left[x_{0}\right] \widetilde{G}$. Since $f$ is a probabilistic $G$-contraction and $\left(f^{n_{k}} x_{0}, \varrho\right) \in E(G)$ for all $k \geq N$. For $t>0$ and $k \geq N$, we get

$$
\begin{aligned}
F_{f^{n} k^{+1} x_{0}, f \varrho}(t) & \geq F_{f^{n} k^{+1} x_{0}, f \varrho}(\alpha t) \\
& \geq F_{f^{n} x_{x_{0}, \varrho}}(t) \rightarrow 1 \quad \text { as } k \rightarrow \infty
\end{aligned}
$$

We obtain

$$
\lim _{k \rightarrow \infty} f^{n_{k}+1} x_{0}=f \varrho
$$

Hence, we conclude that $f \varrho=\varrho$. Now, let $y \in\left[x_{0}\right]_{\widetilde{G}}$, then from Lemma 3.4 we get

$$
\lim _{n \rightarrow \infty} f^{n} y=\varrho
$$

Next to prove the uniqueness of a fixed point, suppose $\varrho^{*} \in\left[x_{0}\right]_{\widetilde{G}}=[\varrho]_{\widetilde{G}}$ such that $f \varrho^{*}=\varrho^{*}$. Then from Lemma 3.4, for $t>0$, we have

$$
F_{\varrho, \varrho^{*}}(t)=F_{f^{n} \varrho, f^{n} \varrho^{*}}(t) \rightarrow 1, \quad n \rightarrow \infty
$$

Hence, $\varrho^{*}=\varrho$. Moreover, if $G$ is weakly connected, then $f$ is a Picard operator as $\left[x_{0}\right]_{\widetilde{G}}=S$.

(ii) Let $G$ be a weakly connected $\left(H_{f}\right)$-graph. By using the same arguments as in the first part of the proof, we obtain $\lim _{n \rightarrow \infty} f^{n} x_{0}=\varrho$. For each $n \in \mathbb{N}$ let $\left(z_{i}^{n}\right) ; i=0, \ldots, M_{n}$ be a path in $\widetilde{G}$ from $f^{n} x_{0}$ to $\varrho$ with $z_{0}^{n}=f^{n} x_{0}, z_{M}^{n}=\varrho$ and $\left(z_{i-1}^{n}, z_{i}^{n}\right) \in E(\widetilde{G})$.

$$
\begin{aligned}
F_{\varrho, f \varrho}(t) & \geq F_{\varrho, f \varrho}(\alpha t) \\
& \geq \Delta\left(F_{\varrho, f^{n+1} x_{0}}\left(\frac{\alpha t}{2}\right), F_{f^{n+1} x_{0} f \varrho}\left(\frac{\alpha t}{2}\right)\right) \\
& \geq \Delta\left(F_{\varrho, f^{n+1} x_{0}}\left(\frac{\alpha t}{2}\right), \Delta_{i=1}^{M_{n}} F_{f z_{i-1}^{n} f z_{i}^{n}}\left(\frac{\alpha t}{2 M_{n}}\right)\right) \\
& \geq \Delta\left(F_{\varrho, f^{n+1} x_{0}}\left(\frac{\alpha t}{2}\right), \Delta_{i=1}^{M_{n}} F_{z_{i-1}^{n}, z_{i}^{n}}\left(\frac{t}{2 M_{n}}\right)\right) \\
& \geq \Delta\left(F_{\varrho, f^{n+1} x_{0}}\left(\frac{\alpha t}{2}\right), L_{f^{n} x_{0}, \varrho}\left(\frac{t}{2 M}\right)\right),
\end{aligned}
$$

where $M=\max \left\{M_{n}: n \in \mathbb{N}\right\}$.

Since $G$ is an $\left(H_{f}\right)$-graph and $\left(f^{n} x_{0}, f^{n+1} x_{0}\right) \in E(G)$ for $n \in \mathbb{N}$ with $\lim _{n \rightarrow \infty} f^{n} x_{0}=\varrho \in S$, then the sequence of functions $\left\{L_{f^{n} x_{0}, \varrho}(t)\right\}$ converges to $\epsilon_{0}(t)(t>0)$ uniformly. Let $t>0$ 
and $\delta>0$ be given. Since the family $\left\{\Delta^{p}(t)\right\}_{p} \in \mathbb{N}$ is equicontinuous at point $t=1$, there exists $\lambda(\delta) \in(0,1)$ such that $\Delta^{p}(1-\lambda)>1-\delta$ for every $p \in \mathbb{N}$. Choose $n_{0} \in \mathbb{N}$ such that for all $n \geq n_{0}$ we have $F_{\varrho, f^{n+1} x_{0}}\left(\frac{\alpha t}{2}\right)>1-\lambda$ and $L_{f^{n} x_{0}, \varrho}\left(\frac{t}{2 M}\right)>1-\lambda$. So that in view of (3.7), for all $n \geq n_{0}$, we have

$$
\begin{aligned}
F_{Q, f \varrho}(t) & \geq \Delta(1-\lambda, 1-\lambda) \\
& =\Delta^{1}(1-\lambda)>1-\delta .
\end{aligned}
$$

Hence, we deduce $f \varrho=\varrho$. Finally, let $y \in S=\left[x_{0}\right]_{\tilde{G}}$ be arbitrary, then from Lemma 3.4, $\lim _{n \rightarrow \infty} f^{n} y=\varrho$.

Corollary 3.10 Let $(S, \mathcal{F}, \Delta)$ be a complete Menger PM-space under a t-norm $\Delta$ of $\mathcal{H}$-type. Assume that $S$ is endowed with a graph $G$ which is either $\left(C_{f}\right)$-graph or $\left(H_{f}\right)$-graph. Then the following statements are equivalent:

(i) $G$ is weakly connected.

(ii) For every probabilistic G-contraction $f$ on $S$, if there exists $x_{0} \in S$ such that $\left(x_{0}, f x_{0}\right) \in E(G)$, then $f$ is a Picard operator.

Proof (i) $\Rightarrow$ (ii): It is immediate from Theorem 3.9.

(ii) $\Rightarrow$ (i): Suppose that $G$ is not weakly connected. Then $\widetilde{G}$ is disconnected, i.e., there exists $x^{*} \in S$ such that $\left[x^{*}\right]_{\tilde{G}} \neq \emptyset$ and $S \backslash\left[x^{*}\right]_{\tilde{G}} \neq \emptyset$. Let $y^{*} \in S \backslash\left[x^{*}\right]_{\tilde{G}}$, we construct a selfmapping $f$ by

$$
f x= \begin{cases}x^{*} & \text { if } x \in\left[x^{*}\right] \widetilde{G}, \\ y^{*} & \text { if } x \in S \backslash\left[x^{*}\right]_{\widetilde{G}} .\end{cases}
$$

Let $(x, y) \in E(G)$, then $[x]_{\widetilde{G}}:=[y]_{\widetilde{G}}$, which implies $f x=f y$. Hence $(f x, f y) \in E(G)$, since $G$ contains all loops. Thus the mapping $f$ preserves edges. Also, for $t>0$ and $\alpha \in(0,1)$, we have $F_{f x, f y}(\alpha t)=1 \geq F_{x, y}(t)$; thus (3.1) is trivially satisfied. But $x^{*}$ and $y^{*}$ are two fixed points of $f$ contradicting the fact that $f$ is a Picard operator.

Remark 3.11 Taking $G=(S, S \times S)$, Theorem 3.9 improves and extends the result of Sehgal [12, Theorem 3] to all Menger PM-spaces with $t$-norms of $\mathcal{H}$-type. Theorem 3.9 generalizes claim $4^{0}$ of [8, Theorem 3.2], and thus we have the following consequence.

Corollary 3.12 (Jachymski [8, Theorem 3.2]) Let $(S, d)$ be a complete metric space endowed with the graph $G$. Assume that the mapping $f: S \rightarrow S$ is a Banach G-contraction and the following property is satisfied:

$(\mathcal{P})$ For any sequence $\left\{x_{n}\right\}$ in $S$, if $x_{n} \rightarrow x$ in $S$ and $\left(x_{n}, x_{n+1}\right) \in E(G)$ for all $n \geq 1$, then there exists a subsequence $\left\{x_{n_{k}}\right\}$ with $\left(x_{n_{k}}, x\right) \in E(G)$ for all $k \geq 1$.

If there exists $x_{0} \in S$ with $\left(x_{0}, f x_{0}\right) \in E(G)$, then $\left.f\right|_{\left[x_{0}\right]_{\tilde{G}}}$ is a Picard operator. Furthermore, if $G$ is weakly connected, then $f$ is a Picard operator.

Proof Let $\left(S, \mathcal{F}, \Delta_{M}\right)$ be the Menger PM-space induced by the metric $d$. Since the mapping $f$ is a Banach $G$-contraction, then it is a probabilistic G-contraction (see Example 3.2) 
and property $(\mathcal{P})$ invokes that $G$ is a $\left(C_{f}\right)$-graph. Hence the conclusion follows from Theorem 3.9(i).

Example 3.13 Let $\left(X, \mathcal{F}, \Delta_{M}\right)$ be a Menger PM-space where $X=[0, \infty)$ and $F_{x, y}(t)=\frac{t}{t+|x-y|}$ for $t>0$. Then $\left(X, \mathcal{F}, \Delta_{M}\right)$ is complete. Define a self-mapping $f$ on $X$ by

$$
f x= \begin{cases}\frac{x^{2}}{p} & \text { if } x=\frac{1}{n} \text { and } p \geq 3 \text { is a fixed integer } \\ 0 & \text { otherwise. }\end{cases}
$$

Further assume that $X$ is endowed with a graph $G$ consisting of $V(G):=X$ and $E(G):=$ $\Omega \cup\left\{\left(\frac{1}{n}, \frac{1}{m}\right): n, m \in \mathbb{N}\right.$ and $\left.n \mid m\right\} \cup\left\{(x, 0): x \neq \frac{1}{n}\right\}$. It can be seen that $f$ is a probabilistic $G$-contraction with $\alpha=\frac{2}{p}$ and satisfies all the conditions of Theorem 3.9(i).

Note that for $x=1$ and $y=\frac{5}{6}$ and for each $\alpha \in(0,1)$, we can easily set $0<t<\frac{1}{6(1-\alpha)}$ such that

$$
\frac{\alpha t}{\alpha t+\left|\frac{1}{3}-0\right|}<\frac{t}{t+\left|1-\frac{5}{6}\right|}
$$

or

$$
F_{f 1, \frac{5}{6}}(\alpha t)<F_{1, \frac{5}{6}}(t) \quad \text { for } 0<t<\frac{1}{6(1-\alpha)} \text {. }
$$

Hence, one cannot invoke [12, Theorem 3].

Definition 3.14 Let $(S, \mathcal{F}, \Delta)$ be a Menger PM-space under a $t$-norm $\Delta$ of $\mathcal{H}$-type. A mapping $f: S \rightarrow S$ is said to be: (i) continuous at point $x \in S$ whenever $x_{n} \rightarrow x$ in $S$ implies $f x_{n} \rightarrow f x$ as $n \rightarrow \infty$; (ii) orbitally continuous if for all $x, y \in S$ and any sequence $\left\{k_{n}\right\}_{n \in \mathbb{N}}$ of positive integers, $f^{k_{n}} x \rightarrow y$ implies $f\left(f^{k_{n}} x\right) \rightarrow f y$ as $n \rightarrow \infty$; (iii) orbitally G-continuous if for all $x, y \in S$ and any sequence $\left\{k_{n}\right\}_{n \in \mathbb{N}}$ of positive integers, $f^{k_{n}} x \rightarrow y$ and $\left(f^{k_{n}} x, f^{k_{n}+1} x\right) \in$ $E(G) \forall n \in \mathbb{N}$ imply $f\left(f^{k_{n}} x\right) \rightarrow f y$ (see [8]).

Theorem 3.15 Let $(S, \mathcal{F}, \Delta)$ be a complete Menger PM-space under a $t$-norm $\Delta$ of $\mathcal{H}$-type. Assume that the mapping $f: S \rightarrow S$ is a probabilistic G-contraction such that $f$ is orbitally G-continuous, and let there exist $x_{0} \in S$ such that $\left(x_{0}, f x_{0}\right) \in E(G)$. Then $f$ has a unique fixed point $\varrho \in S$ and for every $y \in\left[x_{0}\right]_{\widetilde{G}}, f^{n} y \rightarrow \varrho$. Moreover, if $G$ is weakly connected, then $f$ is a Picard operator.

Proof Let $\left(x_{0}, f x_{0}\right) \in E(G)$, by induction $\left(f^{n} x_{0}, f^{n+1} x_{0}\right) \in E(G)$ for all $n \in \mathbb{N}$. By using Lemma 3.8 , it follows that $f^{n} x_{0} \rightarrow \varrho \in S$. Since $f$ is orbitally G-continuous, then $f\left(f^{n} x_{0}\right) \rightarrow f \varrho$. This gives $\varrho=f \varrho$. From Lemma 3.4 for any $y \in\left[x_{0}\right] \widetilde{G}_{\tilde{G}}, f^{n} y \rightarrow \varrho$.

Remark 3.16 We note that in Theorem 3.15 the assumption that $f$ is orbitally Gcontinuous can be replaced by orbital continuity or continuity of $f$.

Remark 3.17 Theorem 3.15 generalizes and extends claims $2^{0}$ and $3^{0}$ [8, Theorem 3.3] and claim $3^{0}[8$, Theorem 3.4]. 
As a consequence of Theorems 3.9 and 3.15, we obtain the following corollary, which is actually a probabilistic version of Theorem 1.1 and thus generalizes and extends the results of Nieto and Rodríguez-López [4, Theorems 2.1 and 2.3], Petruşel and Rus [3, Theorem 4.3] and Ran and Reurings [2, Theorem 2.1].

Corollary 3.18 Let $(S, \preceq)$ be a partially ordered set, and let $(S, \mathcal{F}, \Delta)$ be a complete Menger PM-space under a $t$-norm $\Delta$ of $\mathcal{H}$-type. Assume that the mapping $f: S \rightarrow S$ is nondecreasing (nonincreasing) with respect to the order '

$$
F_{f x, f y}(\alpha t) \geq F_{x, y}(t) \quad \text { for all } x, y \in S, x \preceq y(t>0) .
$$

\section{Also suppose that either}

(i) $f$ is continuous, or

(ii) for every nondecreasing sequence $\left\{x_{n}\right\}$ in $S$ such that $x_{n} \rightarrow x$ in $S$, we have $x_{n} \preceq x$ for all $n \geq 1$.

If there exists $x_{0} \in S$ with $x_{0} \preceq f x_{0}$, then $f$ has a fixed point. Furthermore, if $(S, \preceq)$ is such that every pair of elements of $S$ has an upper or lower bound, then $f$ is a Picard operator.

Proof Consider a graph $G_{1}$ consisting of $V\left(G_{1}\right)=S$ and $E\left(G_{1}\right)=\{(x, y) \in S \times S: x \preceq y\}$. If $f$ is nondecreasing, then it preserves edges w.r.t. graph $G_{1}$ and condition (3.10) becomes equivalent to (3.1). Thus $f$ is a probabilistic $G_{1}$-contraction. In case $f$ is nonincreasing, consider $G_{2}$ with $E\left(G_{2}\right)=\{(x, y) \in S \times S: x \preceq y$ or $x \succeq y\}$ and a vertex set coincides with $S$. Actually, $G_{2}:=\widetilde{G_{1}}$ and from Proposition 3.3 if $f$ is a probabilistic $G_{1}$-contraction, then it is a probabilistic $G_{2}$ contraction. Now if $f$ is continuous, then the conclusion follows from Theorem 3.15. On the other hand, if (ii) holds, then $G_{1}$ and $G_{2}$ are $\left(C_{f}\right)$-graphs and conclusions follow from the first part of Theorem 3.9.

By relaxing $\mathcal{H}$-type condition on a $t$-norm, our next result deals with a compact Menger PM-space using the following class of graphs as the fixed point property is closely related to the connectivity of a graph.

Definition 3.19 Let $(S, \mathcal{F})$ be a PM-space endowed with a graph $G$ and $f: S \rightarrow S$. Assume the sequence $\left\{f^{n} x\right\}$ in $S$ with $\left(f^{n} x, f^{n+1} x\right) \in E(G)$ for $n \in \mathbb{N}$ and $F_{f^{n} x_{f} f^{n+1} x}(t) \rightarrow 1(t>0)$, we say that the graph $G$ is $\left(E_{f}\right)$-graph if for any subsequence $f^{n_{k}} x \rightarrow z \in S$, there exists a natural number $N$ such that $\left(f^{n_{k}} x, z\right) \in E(G)$ for all $k \geq N$.

Theorem 3.20 Let $(S, \mathcal{F}, \Delta)$ be a compact Menger PM-space under a t-norm $\Delta$ satisfying $\sup _{a<1} \Delta(a, a)=1$. Assume that the mapping $f: S \rightarrow S$ is a probabilistic G-contraction, and let there exist $x_{0} \in S$ such that $\left(x_{0}, f x_{0}\right) \in E(G)$. If $G$ is an $\left(E_{f}\right)$-graph, then $f$ has a unique fixed point $\varrho \in\left[x_{0}\right] \widetilde{G}$.

Proof Since $\left(x_{0}, f x_{0}\right) \in E(G)$, then $\left(f^{n} x_{0}, f^{n+1} x_{0}\right) \in E(G)$ for $n \in \mathbb{N}$ and

$$
\begin{aligned}
F_{f^{n} x_{0}, f^{n+1} x_{0}}(t) & \geq F_{f^{n-1} x_{0}, f^{n} x_{0}}\left(\frac{t}{\alpha}\right) \\
& \cdots \\
& \geq F_{x_{0}, f x_{0}}\left(\frac{t}{\alpha^{n}}\right) \rightarrow 1 \quad \text { as } n \rightarrow \infty(t>0) .
\end{aligned}
$$


From compactness, let $\left\{f^{n_{k}} x_{0}\right\}$ be a subsequence such that $f^{n_{k}} x_{0} \rightarrow \varrho \in S$. Let $t>0$ and $\delta>$ 0 be given. Since $\sup _{a<1} \Delta(a, a)=1$, then there exists $\lambda(\delta) \in(0,1)$ such that $\Delta(1-\lambda, 1-\lambda)>$ $1-\delta$ choose $n^{\prime} \in \mathbb{N}$ such that for all $k \geq n^{\prime}$ we have $F_{f^{n} k_{x_{0}, \varrho}}\left(\frac{t}{2}\right)>1-\lambda$ and $F_{f^{n} k_{x_{0}} f^{n_{k}+1} x_{0}}\left(\frac{t}{2}\right)>$ $1-\lambda$. Then we obtain

$$
\begin{aligned}
F_{f^{n_{k}+1} x_{0}, \varrho}(t) & \geq \Delta\left(F_{f^{n} k^{+1} x_{0}, f^{n} k_{x_{0}}}\left(\frac{t}{2}\right), F_{f^{n} k_{x_{0}, \varrho}}\left(\frac{t}{2}\right)\right) \\
& \geq \Delta(1-\lambda, 1-\lambda)>1-\delta .
\end{aligned}
$$

Thus, $f^{n_{k}+1} x_{0} \rightarrow \varrho$.

Choose $n_{1} \in \mathbb{N}$ such that for all $k \geq n_{1}$ we have $F_{f^{n} k^{+1} x_{0}, \varrho}\left(\frac{t}{2}\right)>1-\lambda$ and $F_{f^{n} k_{x_{0}, \varrho}}\left(\frac{t}{2 \alpha}\right)>1-\lambda$. Since $G$ is an $\left(E_{f}\right)$-graph, there exists $n_{2} \in \mathbb{N}$ such that $\left(f^{n_{k}} x_{0}, \varrho\right) \in E(G)$ for all $k \geq n_{2}$. Let $n_{0}=\max \left\{n_{1}, n_{2}\right\}$, then for $k \geq n_{0}$ we get

$$
\begin{aligned}
F_{f \varrho, \varrho}(t) & \geq \Delta\left(F_{f^{n_{k}+1} x_{0}, f \varrho}\left(\frac{t}{2}\right), F_{f^{n^{k}+1} x_{0}, \varrho}\left(\frac{t}{2}\right)\right) \\
& \geq \Delta\left(F_{f^{n_{k} x_{0}, \varrho}}\left(\frac{t}{2 \alpha}\right), F_{f^{n_{k}+1} x_{0}, \varrho}\left(\frac{t}{2}\right)\right) \\
& \geq \Delta(1-\lambda, 1-\lambda)>1-\delta .
\end{aligned}
$$

Hence, $f \varrho=\varrho$. Note that $\left\{x_{0}, f x_{0}, \ldots, f^{n_{1}} x_{0}, \ldots, f^{n_{N}} x_{0}, \varrho\right\}$ is a path in $\widetilde{G}$, so that $\varrho \in\left[x_{0}\right] \widetilde{G}$.

So far it remains to investigate whether Theorem 3.20 can be extended to a complete PM-space?

Definition 3.21 [12] Let $(S, \mathcal{F})$ be a PM-space, and let $\varepsilon>0$ and $0<\delta<1$ be fixed real numbers. A mapping $f: S \rightarrow S$ is said to be $(\varepsilon, \delta)$-contraction if there exists a constant $\alpha \in(0,1)$ such that for $x \in S$ and $y \in \mathcal{N}_{x}(\varepsilon, \delta)$ we have

$$
F_{f x, f y}(\alpha t) \geq F_{x, y}(t) \text { for all } t>0 \text {. }
$$

The PM space $(S, \mathcal{F})$ is said to be $(\varepsilon, \delta)$-chainable if for each $x, y \in S$ there exists a finite sequence $\left(x_{n}\right)_{n=0}^{N}$ of elements in $S$ with $x_{0}=x$ and $x_{N}=y$ such that $x_{i+1} \in \mathcal{N}_{x_{i}}(\varepsilon, \delta)$ for $i=$ $0,1, \ldots, N-1$.

It is important to note that every $(\varepsilon, \delta)$-contraction mapping is continuous. Let $x_{n} \rightarrow x$ in $S$, then there exists a natural number $N(\varepsilon, \delta)$ such that $x_{n} \in \mathcal{N}_{x}(\varepsilon, \delta)$ for all $n \geq N$. Thus, for $t>0$ and for all $n \geq N$, we obtain

$$
\begin{aligned}
F_{f x_{n}, f x}(t) & \geq F_{f x_{n}, f x}(\alpha t) \\
& \geq F_{x_{n}, x}(t) \rightarrow 1 \quad \text { as } n \rightarrow \infty .
\end{aligned}
$$

Hence, $f x_{n} \rightarrow f x$.

Theorem 3.22 Let $(S, \mathcal{F}, \Delta)$ be a complete $(\varepsilon, \delta)$-chainable Menger PM-space under a $t$-norm $\Delta$ of $\mathcal{H}$-type. Let the mapping $f: S \rightarrow S$ be an $(\varepsilon, \delta)$-contraction. Then $f$ is a Picard operator. 
Proof Consider the graph $G$ consisting of $E(G)=\left\{(x, y) \in S \times S: F_{x, y}(\varepsilon)>1-\delta\right\}$ and $V(G)$ coinciding with $S$. Let $x, y \in S$. Since the PM-space $(S, \mathcal{F})$ is $(\varepsilon, \delta)$-chainable, there exists a finite sequence $\left(x_{i}\right)_{i=0}^{N}$ in $S$ with $x_{0}=x$ and $x_{N}=y$ such that $F_{x_{i}, x_{i+1}}(\varepsilon)>1-\delta$ for $i=0,1, \ldots N-1$. Hence, $\left(x_{i}, x_{i+1}\right) \in E(G)$ for $i=0,1, \ldots, N-1$, which yields that $G$ is connected. Let $(x, y) \in E(G)$, then $y \in \mathcal{N}_{x}(\varepsilon, \delta)$. Since the mapping $f$ is an $(\varepsilon, \delta)$-contraction, thus (3.1) is satisfied. Finally we have

$$
\begin{aligned}
F_{f x, f y}(\varepsilon) & \geq F_{f x, f y}(\alpha \varepsilon) \\
& \geq F_{x, y}(\varepsilon)>1-\delta .
\end{aligned}
$$

Thus, $(f x, f y) \in E(G)$. Hence, $f$ is a probabilistic $G$-contraction and the conclusion follows from Theorem 3.15 .

Remark 3.23 Theorem 3.22 has an advantage over Theorem 7 of Sehgal and BharuchaReid [12] which is only restricted to continuous $t$-norms satisfying $\Delta(t, t) \geq t$. Moreover, the proof of our result is rather simple and easy, which invokes the novelty of Theorem 3.22 .

Definition 3.24 (Edelstein $[19,20])$ The metric space $(S, d)$ is $\varepsilon$-chainbale for some $\varepsilon>0$ if for every $x, y \in S$, there exists a finite sequence $\left(x_{i}\right)_{n=0}^{N}$ of elements in $S$ with $x_{0}=x, x_{N}=y$ and $d\left(x_{i}, x_{i+1}\right)<\varepsilon$ for $i=0,1, \ldots, N-1$.

Remark 3.25 [12] If $(S, d)$ is an $\varepsilon$-chainable metric space, then the induced Menger PMspace $\left(S, \mathcal{F}, \Delta_{M}\right)$ is an $(\varepsilon, \delta)$-chainable space.

Corollary 3.26 (Edelstein $[19,20])$ Let $(S, d)$ be a complete $\varepsilon$-chainable metric space. Let $f: S \rightarrow S$ and let there exist $\alpha \in(0,1)$ such that

$$
\forall_{x, y \in S}\{d(x, y)<\varepsilon \Longrightarrow d(f x, f y) \leq \alpha d(x, y)\}
$$

\section{Then $f$ is a Picard operator.}

Proof Since the metric space $(S, d)$ is $\varepsilon$-chainable, then the induced Menger PM-space $(S, \mathcal{F}, \Delta)$ is $(\varepsilon, \delta)$-chainable for each $0<\delta<1$. We only need to show that the self-mapping $f$ on $S$ is an $(\varepsilon, \delta)$-contraction. Let $x, y \in S$ be such that $y \in \mathcal{N}_{x}(\varepsilon, \delta)$, i.e., $F_{x, y}(\varepsilon)>1-\delta$ or $\epsilon_{0}(\varepsilon-d(x, y))>1-\delta$. The definition of $\epsilon_{0}$ implies $d(x, y)<\varepsilon$ and thus $d(f x, f y) \leq \alpha d(x, y)$. Now, for $t>0$, we get

$$
\begin{aligned}
F_{f x, f y}(\alpha t) & =\epsilon_{0}(\alpha t-d(f x, f y)) \\
& \geq \epsilon_{0}(t-d(x, y)) \\
& =F_{x, y}(t) .
\end{aligned}
$$

Hence the conclusion follows from Theorem 3.22.

Kirk et al. [21] introduced the idea of cyclic contractions and established fixed point results for such mappings. 
Let $S$ be a nonempty set, let $m$ be a positive integer, let $\left\{A_{i}\right\}_{i=1}^{m}$ be nonempty closed subsets of $S$, and let $f: \bigcup_{i=1}^{m} A_{i} \rightarrow \bigcup_{i=1}^{m} A_{i}$ be an operator. Then $S:=\bigcup_{i=1}^{m} A_{i}$ is known as a cyclic representation of $S$ w.r.t. $f$ if

$$
f\left(A_{1}\right) \subset A_{2}, \ldots, f\left(A_{m-1}\right) \subset A_{m}, f\left(A_{m}\right) \subset A_{1}
$$

and the operator $f$ is known as a cyclic operator [21].

In the following, we present the probabilistic version of the main result of [21], as a last consequence of Theorem 3.9.

Theorem 3.27 Let $(S, \mathcal{F}, \Delta)$ be a complete Menger PM-space under a $t$-norm $\Delta$ of $\mathcal{H}$ type. Let $m$ be a positive integer, let $\left\{A_{i}\right\}_{i=1}^{m}$ be nonempty closed subsets of $S, Y:=\bigcup_{i=1}^{m} A_{i}$ and $f: Y \rightarrow Y$. Assume that

(i) $\bigcup_{i=1}^{m} A_{i}$ is a cyclic representation of $Y$ w.r.t.f;

(ii) $\exists \alpha \in(0,1)$ such that $d(f x, f y) \leq \alpha d(x, y)$ whenever $x \in A_{i}, y \in A_{i+1}$, where $A_{m+1}=A_{1}$. Then $f$ has a unique fixed point $\varrho \in \bigcap_{i=1}^{m} A_{i}$ and $f^{n} y \rightarrow \varrho$ for any $y \in \bigcup_{i=1}^{m} A_{i}$.

Proof Since $\bigcup_{i=1}^{m} A_{i}$ is closed, then $(Y, \mathcal{F}, \Delta)$ is complete. Let us consider a graph $G$ consisting of $V(G):=Y$ and $E(G):=\Omega \cup\left\{(x, y) \in Y \times Y: x \in A_{i}, y \in A_{i+1} ; i=1, \ldots, m\right\}$. By (i) it follows that $f$ preserves edges. Now, let $f^{n} x \rightarrow x^{*}$ in $Y$ such that $\left(f^{n} x, f^{n+1} x\right) \in E(G)$ for all $n \in \mathbb{N}$. Then by (3.13) it is inferred that the sequence $\left\{f^{n} x\right\}$ has infinitely many terms in each $A_{i} ; i \in\{1,2, \ldots, m\}$. So that one can easily identify a subsequence of $\left\{f^{n} x\right\}$ converging to $x^{*}$ in each $A_{i}$; and since $A_{i}$ 's are closed, then $x^{*} \in \bigcap_{i=1}^{m} A_{i}$. Thus, we can easily form a subsequence $\left\{f^{n_{k}} x\right\}$ in some $A_{j}, j \in\{1, \ldots, m\}$ such that $\left(f^{n_{k}} x, x^{*}\right) \in E(G)$ for $k \geq 1$. It elicits that $G$ is a weakly connected $\left(C_{f}\right)$-graph. Hence, by Theorem 3.9 conclusion follows.

\section{Competing interests}

The authors declare that they have no competing interests.

Authors' contributions

All authors contributed equally and significantly in writing this paper. All authors read and approved the final manuscript.

\section{Author details}

${ }^{1}$ Department of Mathematics, Quaid-i-Azam University, Islamabad, Pakistan. ${ }^{2}$ Centre for Advanced Mathematics and Physics, National University of Sciences and Technology, H-12, Islamabad, Pakistan. ${ }^{3}$ Department of Mathematics, King Abdulaziz University, Jeddah, Saudi Arabia.

\section{Acknowledgements}

The research of N. Shahzad was partially supported by the Deanship of Scientific Research (DSR), King Abdulaziz University, Jeddah, Saudi Arabia.

Received: 31 May 2013 Accepted: 5 August 2013 Published: 22 August 2013

\section{References}

1. Nieto, JJ, Rodríguez-López, R: Existence and uniqueness of fixed point in partially ordered sets and applications to ordinary differential equations. Acta Math. Sin. Engl. Ser. 23, 2205-2212 (2007)

2. Ran, ACM, Reurings, MCB: A fixed point theorem in partially ordered sets and some applications to matrix equations. Proc. Am. Math. Soc. 132, 1435-1443 (2004)

3. Petrusel, A, Rus, IA: Fixed point theorems in ordered L-spaces. Proc. Am. Math. Soc. 134, 411-418 (2006)

4. Nieto, JJ, Rodríguez-López, R: Contractive mapping theorems in partially ordered sets and applications to ordinary differential equations. Order 22, 223-239 (2005)

5. O'Regan, D, Petruşel, A: Fixed point theorems for generalized contractions in ordered metric spaces. J. Math. Anal. Appl. 341, 1241-1252 (2008)

6. Agarwal, RP, El-Gebeily, MA, O’Regan, E: Generalized contraction in partially ordered metric spaces. Appl. Anal. 87, 1-8 (2008) 
7. Gnana Bhaskar, T, Lakshmikantham, V: Fixed point theorems in partially ordered metric spaces and applications. Nonlinear Anal. 65, 1379-1393 (2006)

8. Jachymski, J: The contraction principle for mappings on a metric space with a graph. Proc. Am. Math. Soc. 136, 1359-1373 (2007)

9. Bojor, F: Fixed point theorems for Reich type contractions on metric spaces with a graph. Nonlinear Anal. 75, 3895-3901 (2012)

10. Samreen, M, Kamran, T: Fixed point theorems for integral G-contractions. Fixed Point Theory Appl. 2013, Article ID 149 (2013)

11. Aleomraninejad, SMA, Rezapour, S, Shahzad, N: Some fixed point results on a metric space with a graph. Topol. Appl. $159,659-663(2012)$

12. Sehgal, VM, Bharucha-Reid, AT: Fixed points of contraction mappings on probabilistic metric spaces. Math. Syst. Theory 6, 97-102 (1972)

13. Schweizer, B, Sklar, A: Probabilistic Metric Spaces. Elsevier/North-Holland, New York (1983)

14. Hadzić, O, Pap, E: Fixed Point Theory in Probabilistic Metric Spaces. Kluwer Academic, Dordrecht (2001)

15. Cho, Y, Park, KS, Chang, SS: Fixed point theorems in metric spaces and probabilistic metric spaces. Int. J. Math. Math. Sci. 19, 243-252 (1996)

16. Hadzić, O: Fixed point theorems for multivalued mapping in probabilistic metric spaces. Fuzzy Sets Syst. 88, $219-226$ (1997)

17. Schweizer, B, Sklar, A, Thorp, E: The metrization of statistical metric spaces. Pac. J. Math. 10,673-675 (1960)

18. Miheț, D: A generalization of a contraction principle in probabilistic metric spaces. Part II. Int. J. Math. Math. Sci. 5 729-736 (2005)

19. Edelstein, M: On fixed and periodic points under contractive mappings. J. Lond. Math. Soc. 37, 74-79 (1962)

20. Edelstein, M: An extension of Banach's contraction principle. Proc. Am. Math. Soc. 12, 7-10 (1961)

21. Kirk, WA, Srinivasan, PS, Veeranmani, P: Fixed points for mappings satisfying cyclical contractive condition. Fixed Point Theory 4(1), 79-89 (2003)

doi:10.1186/1687-1812-2013-223

Cite this article as: Kamran et al.: Probabilistic G-contractions. Fixed Point Theory and Applications 2013 2013:223.

\section{Submit your manuscript to a SpringerOpen ${ }^{\circ}$ journal and benefit from:}

- Convenient online submission

Rigorous peer review

- Immediate publication on acceptance

- Open access: articles freely available online

- High visibility within the field

- Retaining the copyright to your article 